\title{
Comparison of IEEE802.16e and IEEE802.11n in QPSK with LDPC Minimum Sum Algorithm
}

\author{
Yihua Chen, Huating Syu and Zongyi Saio \\ Oriental Institute of Technology, Institute of Information and Communication Engineering, New Taipei City, Taiwan
}

\begin{abstract}
In accordance with the approximate lower triangular parity check matrix standard of low-density parity-check codes in IEEE Standard 802.16e, this study used LabVIEW to write a variety of adjustable encoding patterns generated at the transmitter end within a single-program structure, including six groups of parity check matrices that were created using the four coding rates in 802.16e, and 114 codewords that were formed by developing 19 types of subblock sizes. A decoder with a minimum sum algorithm was employed to examine the structures of nodes and variable nodes based on changes in the selected standards and complete decoding. This paper describes an encoder-decoder mechanism for combining low-density parity-check codes and minimum sum algorithms, and a quadrature phase-shift keying-channel that was developed and applied to the encoderdecoder mechanism to analyze the resulting bit error rate (BER) curves. The BER curve analyses in 802.16e and 802.11n have revealed that the effect of subblock size on the BER was insignificant, and the two standards exhibited the most similar BERs at the code rates of $5 / 6$ and $2 / 3$; however, the error correction achieved by $802.11 \mathrm{n}$ at the code rate of $1 / 2$ was the most effective.
\end{abstract}

Keywords-approximate lower triangulation (ALT); low-density parity-check code (LDPC); subblock size; minimum sum algorithm (MSA)

\section{INTRODUCTION}

In 1948, Claudee Shannon proposed Shannon theory [1], which posits that channel-coding original information bits within the data rate $R_{b}$ and the channel capacity $C$ effectively reduces the bit error rate (BER) of data transmitted through a channel. The longer a codeword is, the closer the BER data are to infinitesimal; this limit is called the Shannon Limit [2]. Currently, numerous error correction codes [3] have been researched, and the low-density parity-check (LDPC) codes proposed by Gallager [4] have been the most extensively studied. Although the encoding-decoding algorithm of LDPC codes is complex, LDPC codes are a type of error correction code with a data transmission rate that is the closest to the Shannon Limit channel capacity. In the present study, approximate lower triangular (ALT) parity check matrices were combined with the system structure depicted in Fig. 1 to encode information bits and then modulate them by using quadrature phase-shift keying (QPSK) or 16-quadrature amplitude modulation (QAM). The bits were transmitted through additive white Gaussian noise (AWGN) to a receiver, in which the bits were demodulated correspondingly. Finally, the original bits were decoded using a minimum sum algorithm (MSA) [5].

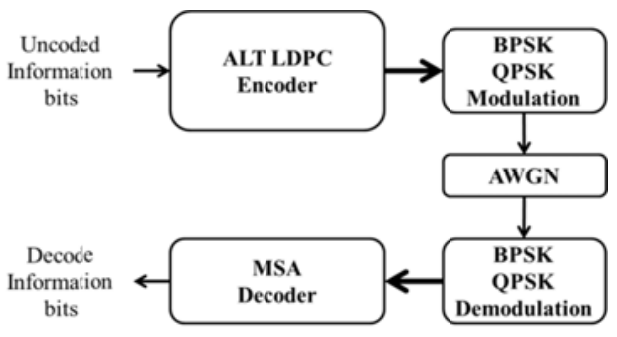

FIGURE I. SCHEMATIC DIAGRAM OF THE ENCODING AND DECODING SYSTEM

The MSA adopted in this study was based on the simplified sum-product algorithm (SPA) [6]. The MSA differs from the SPA in that complex algorithms are not required, thereby accelerating the decoding process.

This study adopted the MSA decoding algorithm used in the ALT encoding standard in the irregular LDPC codes in the 802.16e wireless standards [7]. From the 802.16e standards, four types of code rate were derived to create six groups of parity-check matrices, which were expanded using 19 subblock sizes to form 114 codewords. In this study, the approach used to generate these 114 codewords and the corresponding decoding algorithms were all employed in the program, and the BER curves of a number of specified algorithms were selected for investigation.

\section{APPROXIMATE LOWER TRIANGULAR LDPC ENCODER}

\section{A. ALT LDPC Parity Check Matrix Structures}

LDPC codes are a type of linear block code. Encoding linear block codes typically involves calculating information bit vectors and generating matrices to acquire redundancy bits, which are then added with the information bits to obtain codewords. Decoding involves using the parity check matrices and codeword algorithms to correct errors.

Unlike typical linear block codes, LDPC codes require parity check matrices alone to complete the encoding and decoding process. This study was conducted to improve the encoding approach used in the 802.11n wireless ALT LDPC standard [8], and the same approach was used to accomplish the 802.16e standard encoder. The remainder of this section presents the information regarding the two standards of the parity check matrices. 
TABLE I. THE MATRIX SIZES OF THE TWELVE 802.11N ALT LDPC STANDARD PARITY CHECK MATRICES.

\begin{tabular}{|cc|c|c|c|c|}
\hline$Z$ & Code rate & $\mathbf{1 / 2}$ & $\mathbf{2 / 3}$ & $3 / \mathbf{4}$ & $\mathbf{5 / 6}$ \\
\hline $\mathbf{2 7}$ & $324 \times 648$ & $216 \times 648$ & $162 \times 648$ & $108 \times 648$ \\
\hline $\mathbf{5 4}$ & $648 \times 1296$ & $432 \times 1296$ & $324 \times 1296$ & $216 \times 1296$ \\
\hline $\mathbf{8 1}$ & $972 \times 1944$ & $648 \times 1944$ & $486 \times 1944$ & $324 \times 1944$ \\
\hline
\end{tabular}

TABLE II. THE MATRIX SOZES OF THE SIX 802.16E ALT LDPC STANDARD PARITY CHECK MATRICES

\begin{tabular}{|c|c|c|c|c|c|c|}
\hline Code Rate & $\mathbf{1 / 2}$ & $\mathbf{2 / 3 A}$ & $\mathbf{2 / 3 B}$ & $\mathbf{3 / 4 A}$ & $\mathbf{3 / 4 B}$ & $\mathbf{5} / 6$ \\
\hline Matrix Size & $12 \times 24$ & $8 \times 24$ & $8 \times 24$ & $6 \times 24$ & $6 \times 24$ & $4 \times 24$ \\
\hline $\mathbf{2 4}$ & $288 \times 576$ & $192 \times 576$ & $192 \times 576$ & $144 \times 576$ & $144 \times 576$ & $96 \times 576$ \\
\hline $\mathbf{2 8}$ & $336 \times 672$ & $224 \times 672$ & $224 \times 672$ & $168 \times 672$ & $168 \times 672$ & $112 \times 672$ \\
\hline $\mathbf{3 2}$ & $384 \times 768$ & $256 \times 768$ & $256 \times 768$ & $192 \times 768$ & $192 \times 768$ & $128 \times 768$ \\
\hline $\mathbf{3 6}$ & $432 \times 864$ & $288 \times 864$ & $288 \times 864$ & $216 \times 864$ & $216 \times 864$ & $144 \times 864$ \\
\hline $\mathbf{4 0}$ & $480 \times 960$ & $320 \times 960$ & $320 \times 960$ & $240 \times 960$ & $240 \times 960$ & $160 \times 960$ \\
\hline $\mathbf{4 4}$ & $528 \times 1056$ & $352 \times 1056$ & $352 \times 1056$ & $264 \times 1056$ & $264 \times 1056$ & $176 \times 1056$ \\
\hline $\mathbf{4 8}$ & $576 \times 1152$ & $384 \times 1152$ & $384 \times 1152$ & $288 \times 1152$ & $288 \times 1152$ & $192 \times 1152$ \\
\hline $\mathbf{5 2}$ & $624 \times 1248$ & $416 \times 1248$ & $416 \times 1248$ & $312 \times 1248$ & $312 \times 1248$ & $208 \times 1248$ \\
\hline $\mathbf{5 6}$ & $672 \times 1344$ & $448 \times 1344$ & $448 \times 1344$ & $336 \times 1344$ & $336 \times 1344$ & $224 \times 1344$ \\
\hline $\mathbf{6 0}$ & $720 \times 1440$ & $480 \times 1440$ & $480 \times 1440$ & $360 \times 1440$ & $360 \times 1440$ & $240 \times 1440$ \\
\hline $\mathbf{6 4}$ & $768 \times 1536$ & $512 \times 1536$ & $512 \times 1536$ & $384 \times 1536$ & $384 \times 1536$ & $256 \times 1536$ \\
\hline $\mathbf{6 8}$ & $816 \times 1632$ & $544 \times 1632$ & $544 \times 1632$ & $408 \times 1632$ & $408 \times 1632$ & $272 \times 1632$ \\
\hline $\mathbf{7 2}$ & $864 \times 1728$ & $576 \times 1728$ & $576 \times 1728$ & $432 \times 1728$ & $432 \times 1728$ & $288 \times 1728$ \\
\hline $\mathbf{7 6}$ & $912 \times 1824$ & $608 \times 1824$ & $608 \times 1824$ & $456 \times 1824$ & $456 \times 1824$ & $304 \times 1824$ \\
\hline $\mathbf{8 0}$ & $960 \times 1920$ & $640 \times 1920$ & $640 \times 1920$ & $480 \times 1920$ & $480 \times 1920$ & $320 \times 1920$ \\
\hline $\mathbf{8 4}$ & $1008 \times 2016$ & $672 \times 2016$ & $672 \times 2016$ & $504 \times 2016$ & $504 \times 2016$ & $336 \times 2016$ \\
\hline $\mathbf{8 8}$ & $1056 \times 2112$ & $704 \times 2112$ & $704 \times 2112$ & $528 \times 2112$ & $528 \times 2112$ & $352 \times 2112$ \\
\hline $\mathbf{9 2}$ & $1104 \times 2208$ & $736 \times 2208$ & $736 \times 2208$ & $552 \times 2208$ & $552 \times 2208$ & $368 \times 2208$ \\
\hline $\mathbf{9 6}$ & $1152 \times 2304$ & $768 \times 2304$ & $768 \times 2304$ & $576 \times 2304$ & $576 \times 2304$ & $384 \times 2304$ \\
\hline
\end{tabular}

Table I lists the matrix sizes of the 12 parity-check matrices in $802.11 \mathrm{n}$, which were generated by combining three types of $\mathrm{Z}$ and four types of code rate. Specifically, each matrix generated using each set of $Z$ and code rate was unique. Table II lists the matrix sizes of the six primary parity-check matrices in 802.16e. According to the code-rate differentiation, the $\mathrm{Z}$ sizes changed only the postexpansion matrix sizes but not the matrix content.

\section{B. Actualization of the IEEE 802.16e ALT LDPC Parity Check Matrices in LabVIEW}

Proposed by Richardson and Urbanke in 2001 [9], ALT LDPC codes involve dividing the established parity check matrix $H$ into six submatrices $(A, B, C, D, T$, and $E$ ) by using algorithmic equations to obtain the redundancy check bits $p_{1}$ and $p_{2}$, and adding the bits with the original information bit vector $m$ to solve the codeword $X=\left[m p_{1} p_{2}\right]$. The equations of the redundancy check bits $p_{1}$ and $p_{2}$ are shown as follows:

$$
\begin{aligned}
& P_{1}=E T^{-1}\left(A m^{T}\right)+C m^{T} \\
& P_{2}=T^{-1}\left(A m^{T}+B P_{1}^{T}\right)
\end{aligned}
$$

A previous study [8] indicated that fulfilling the IEEE 802.11n wireless ALT LDPC standard involves not only the encoding equations, but also executing the processes, as illustrated in Fig. 2. The processes were adopted to rewrite the 802.16e standard, which was then entered into the LabVIEW platform. Figs. 3 and 4 comprehensively depict the program structure and the packaged Sub VI, respectively.

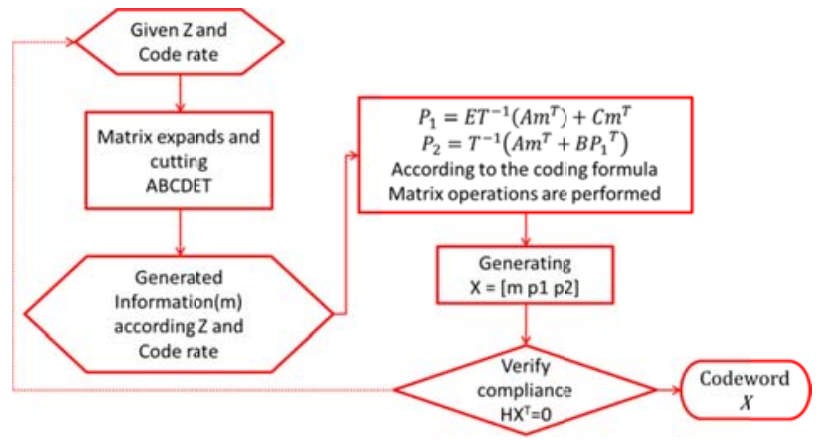

FIGURE II. ALT LDPC ENCODING PROCESS CHART

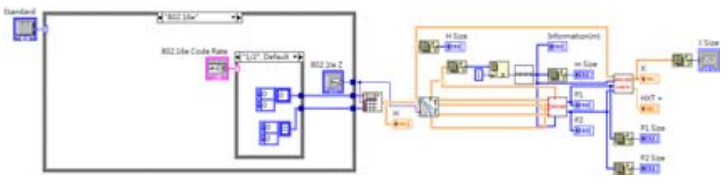

FIGURE III. STRUCTURAL DIAGRAM OF THE ALT LDPC ENCODING PROGRAM

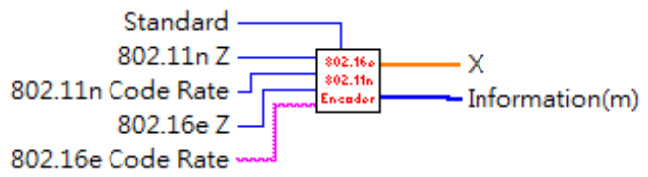

FIGURE IV. COMPREHENSIVE ENCODING PROGRAM SUB VI

In addition to expanding the 802.16e standard, the comprehensive encoding program maintained the 802.11n standard to enable comparing the performance of the decoding algorithms.

\section{Minimum Sum AlgorithM}

Decoding requires using Tanner graphs [10] and converting the numbers of rows and columns of the parity-check matrices to the variable nodes $B(x i)$ and check nodes $C(x i)$. The values 0 and 1 within the matrices were observed, and the variable nodes were connected to the check nodes. Log likelihood ratio (LLR) soft information was transmitted via these connections. However, Table II indicates that within the 802.16e ALT LDPC standard, the sizes of all the check matrices are calculated as percentages. For a detailed description of the decoding approach, the weight $(3,6)$ regular parity-check matrix in [11] was used as a reference [12]. The matrix was employed to describe the method for generating the Tanner graphs and the processes involved in transmitting the LLR information.

Fig. 5 depicts the four aforementioned steps. Step 1 involved initializing the variable nodes, Step 2 involved updating the LLR soft information at the check nodes, and Step 3 involved updating the LLR soft information at the variable nodes. An evaluation was then conducted to determine whether the designated number of iterations had 
been fulfilled. If this condition was met, then the hard decision in Step 4 was executed; otherwise, the LLR soft information values at the variable nodes in Step 3 were recalculated at Step 2 in the subsequent iteration until the designated number of iterations was fulfilled. The detail procedure for implementing the MSA circuit by using LabVIEW is demonstrated clearly in Y. H., Chen; et. Al [12].

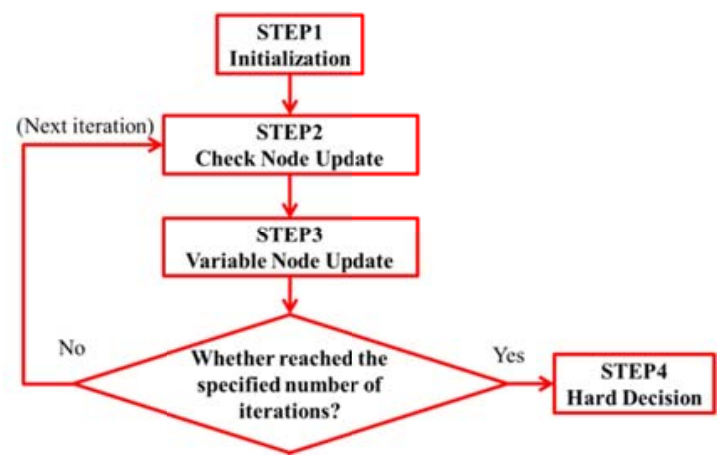

FIGURE V. FLOWCHART OF THE DECODING PROCESS

\section{IEEE 802.11N ALT LDPC STANDARD BER PERFORMANCE ANALYSIS}

LabVIEW was employed to simulate the AWGN channel environment with an Eb/No of $0-1 \mathrm{~dB}$. Each $1 \mathrm{~dB}$ interval generated $81 \mathrm{Mb}$ of random data. Table III shows the corresponding relationship between the $\mathrm{Eb} / \mathrm{No}$ and the $\sigma$. The code rates of $1 / 2,2 / 3 \mathrm{~A}$, and 5/6 were employed to complete the encoding and decoding process, and QPSK was applied to perform the modulation. Decoding calculation was performed using the MSA iteration.

TABLE III. COMPARISON TABLE OF EB/NO VS.

\begin{tabular}{|c|c|c|c|c|c|c|c|c|c|c|c|}
\hline $\mathrm{Eb} / \mathrm{No}, \mathrm{dB}$ & 0 & 1 & 2 & 3 & 4 & 5 & 6 & 7 & 8 & 9 & 10 \\
\hline$\sigma$ & 0.707 & 0.630 & 0.562 & 0.501 & 0.446 & 0.398 & 0.354 & 0.316 & 0.282 & 0.251 & 0.224 \\
\hline
\end{tabular}

First, a comparison of the BER curves of $\mathrm{Z}=24$ bits and $Z=24$ bits, both of which exhibited code rates of 5/6, revealed that the curves were nearly entirely overlapped with each other (Fig. 6), indicating that the subblock sizes did not affect the BERs, and that if the code rates were identical, the BERs were nearly identical. However, only the AWGN channel was simulated in this study, and the subblock sizes affected the codeword lengths. Investigating more complex channel environments, such as Rayleigh fading or burst errors, may yield a more comprehensive discourse.

The aforementioned three decoded BER curves obtained from the receiver calculation were compared separately with the BER curve calculated within the 802.11n standard at the same code rate (Figs. 7 and 8 ), revealing that at the code rates of $5 / 6$ and $2 / 3$, the BER curves of 802.11 n and 802.16e were nearly identical (Fig. 7). By the standard of the BER rate of $10^{-5}$, the coding gain difference was $0.35 \mathrm{~dB}$ at the code rate of $5 / 6$ and $<0.1 \mathrm{~dB}$ at the code rate of $2 / 3$.

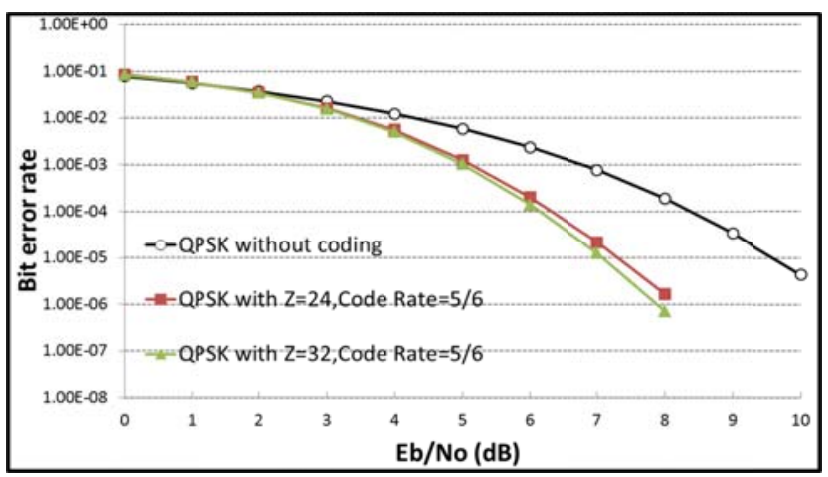

FIGURE VI. COMPARISON OF THE 802.16e BER CURVES AT THE SUBBLOCK SIZE OF 24 AND 32 AND THE CODE RATE OF $5 / 6$

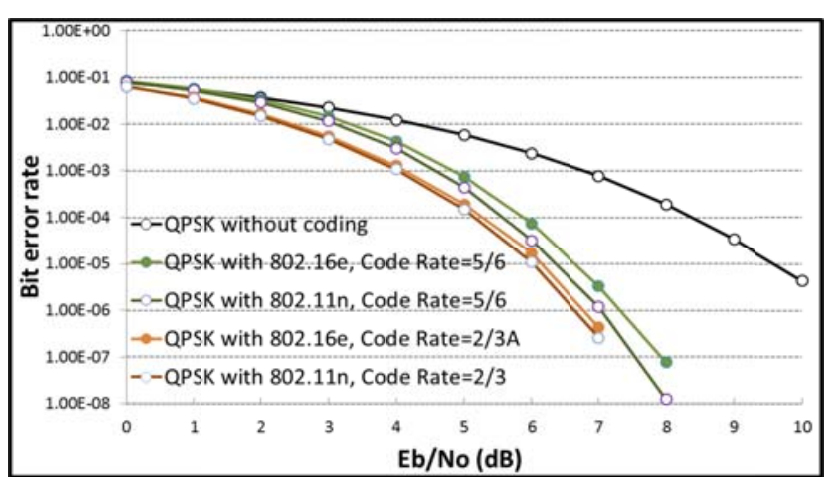

FIGURE VII. COMPARISON OF THE BER CURVES WITH THE 802.16e AND 802.11n CODING AT THE CODE RATES OF 2/3 AND 5/6 TO THE BER CURVE WITHOUT ERROR CORRECTION CODING IN THE LABVIEW SIMULATION INVOLVING THE QPSK MODULATION THROUGH THE AWGN CHANNEL.

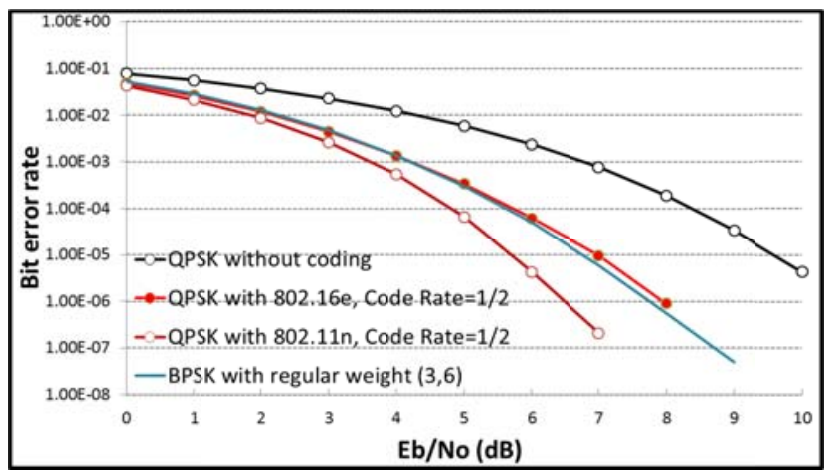

FIGURE VIII. COMPARISON OF THE BER EFFICIENCY WITH THE LDPC CODERS AND DECODERS 802.11n AND 802.16e AT THE CODE RATE OF 1/2 AND THE EFFICIENCY WITH THE REGULAR WEIGHT OF $(3,6)$

Finally, the BER curves of $802.16 \mathrm{e}$ and $802.11 \mathrm{n}$ at the code rate of $1 / 2$ were compared to the BER curve with the regular weight of $(3,6)$ from [11] and [13] (Fig. 8). Although both 802.16 e and 802.11 n were irregular, 802.11n exhibited improved decoding performance. An Eb/No of only $5.7 \mathrm{~dB}$ was required for $802.11 \mathrm{n}$ to reduce the BER to $10^{-5}$, but 802.16e and regular weight $(3,6)$, which exhibited more identical BER curves, required an Eb/No of $7 \mathrm{~dB}$ to reduce 
the BER to $10^{-5}$. Thus, the coding gain difference between 802.16e and $802.11 \mathrm{n}$ at the code rate of $1 / 2$ was $1.3 \mathrm{~dB}$.

\section{CONCLUSION}

In this study, a diversified encoder was created using LabVIEW and the IEEE Standard 802.16e irregular parity-check matrices, and the diversified decoder was completed using the MSA. The BER curve diagrams obtained from simulating the AWGN channel with QPSK modulation were compared to the BER curves obtained from previous studies, revealing that the subblock sizes did not affect the BER in the AWGN channel environment. At the code rates of 5/6 and 2/3, the BERs of 80216e and 802.11n were nearly identical. At the BER of $10^{-5}$, the coding gain difference was only $0.1 \mathrm{~dB}$; however, at the code rate of $1 / 2$ and the BER of $10^{-5}$, the coding gain difference was as high as $1.3 \mathrm{~dB}$. These confirmed that $802.11 \mathrm{n}$ exhibited superior error correction ability compared with 802.16e at the code rate of $1 / 2$, and the error correction ability of $802.16 \mathrm{e}$ was closer to that of the regular LDPC codes at the code rate of $1 / 2$.

Future studies should consider applying the sum-of-product algorithm or forward-backward algorithm [14], [15] to accomplish decoding. In addition, Rayleigh fading or Ricean fading may be implemented in the channels for further investigation of the changes in BER under various scenarios.

\section{REFERENCES}

[1] C. E. Shannon, “A Mathematical Theory of Communication,” Bell Syst. Tech. J, pp. 379-423(Part1);pp. 623-56(Part2), July 1948.

[2] Berrou, C, Glavieux, A,. and Thitimajshima, P., "Near Shannon limit error- correcting coding and decoding: Trubo-codes," IEEE International Conference on Communications, ICC'93, Geneva. Vol. 2, 1993, pp. 1064-1070. May. 1993.

[3] Brenard Sklar, "Digital Communications Fundamentals and Applications SECOND EDITION”.

[4] R. G. Gallager, “Low-Density Parity-Check Codes, ”IRE Trans. Inform. Theroy, pp. 21-28, Jan. 1962.

[5] Jinghu Chen, Marc P. C., ”Near Optimum Universal Belief Propagation Based Decoding of Low-Density Parity Check Codes", IEEE Trans Commun., vol. 50, no. 3, pp. 406-414, Mar. 2002.

[6] F. R. Kschischang, B. J. Frey, and H.-A. Loeliger, "Factor graphs and the sum-product algorithm, ”IEEE Trans. Inform. Theory, VOL. 47. pp. 498-519, Feb. 2001.

[7] IEEE P802.11n?/D1.04 Draft Amendment to STANDARD for Information Technology-Telecommunications and information exchange

[8] Yi Hua Chen, Jue Hsuan Hsiao, Zong Yi Siao, "Wi-Fi LDPC Encoder with Approximate Lower Triangular Diverse Implementation and Verification”, Multi-Conference on Systems, Signals \& Devices (SSD), 978-1-4799-3866-7/14/\$31.00 (C2014 IEEE , pp. 1-6, 2014SSD.

[9] Richardson, T. J, and Urbanke, R. "Efficient encoding of low-density parity-Check Codes, ”IEEE Trans. Inf. Theory, 47. (2), pp. 638-656, 2001.

[10] R. MICHAEL TANNER,”A Recursive Approach to Low Complexity Codes”, IEEE Transactions on information theory, VOL.IT-27, No.5, pp. 533-547 ,September 1981.

[11] Yi-Hua Chen, Chang-Lueng Chu, Jheng-Shyuan He,’FPGA Implementation and Verification of LDPC Minimum Sum Algorithm Decoder with Weight (3, 6) Regular Parity Check Matrix”, ICEMI'2013. pp.682-686. Aug. 2013.
[12] Yi Hua Chen, Jue Hsuan Hsiao, Zong Yi Saio, and Hua Ting Syu , "Minimum Sum Algorithm Decoder for LDPC Nonregular Parity Check Matrix in BPSK System.” PIERS Proceedings, 2136 - 2144, July 6-9, Prague, 2015.

[13] He, Jheng-Shyuan,'Implementation of LDPC Encoder and Decoder on SDR wireless communication system", Thesis-of-Master-degree, O.I.T Institute of information and communication Engineer, July, 2013.

[14] M. Fossocier, M. Mihaljecvic, H. Imai, "Reduced complexity decoding of low-density parity check codes based on belief propagation, ”IEEE Trans. On Commun., VOL. 47 no. 5, pp. 673-680 May 1999.

[15] L. R. Bahl, J. Cocke, F. Jelinek, and J. Raviv, “Optimal decoding of linear codes for minimizing symbol error rate, ”IEEE Trans. Inform. Theory, VOL. 20, pp. 284-287, Mar. 1974. 